\section{Response to Biesecker}

To the Editor: We appreciate the opportunity to respond to the Letter to the Editor by Dr Biesecker ${ }^{1}$ regarding our article reporting the frequency and spectrum of secondary findings in 196 Korean exomes. ${ }^{2}$ Dr Biesecker raises the issue of "overcalling secondary findings," which suggests that $~ 7 \%$ of secondary findings in Koreans are overinflated estimates compared with $1.1-2.0 \%$ of those in Europeans/Africans. Although we could not completely rule out the possibility of overestimation, we sought to avoid this issue by studying two separate groups comprising 100 exomes from healthy controls (group A) and 96 exomes from patients with any kind of underlying disorder (group B). Interestingly, the two groups yielded similar estimates of secondary findings: 7.0\% (7/100) in group A and 6.3\% (6/96) in group B. Nevertheless, the comments by Dr Biesecker, including confidence intervals, provide a useful opportunity to check and refine our standards of interpretation.

Dr Biesecker ${ }^{1}$ suggests that the novel RYR1 truncating variant c.5701C > T (p.Gln1901*) should not have been included as a pathogenic secondary finding in our report. The malignant hyperthermia phenotype is almost entirely associated with missense mutations, and RYR1 mutations are categorized as gain-of-function mutations. Therefore, we may agree with Dr Biesecker's comments, although a few truncating mutations in RYR1 have been reported to cause malignant hyperthermia. ${ }^{3}$ According to the most recent set of consensus guidelines from the American College of Medical Genetics and Genomics and the Association for Molecular Pathology, a truncating variant is expected to be pathogenic only when the known disease mechanism is loss of function. ${ }^{4}$ Therefore, the novel RYR1 truncating variant could be reclassified as "uncertain significance" rather than pathogenic or likely pathogenic.

Dr Biesecker ${ }^{1}$ also indicates that the pathogenicity of a known MUTYH truncating variant, c.799C >T (p.Gln267*), should be reconsidered. We agree that the variant is listed by the Human Gene Mutation Database (HGMD) as "DM?" and that the primary paper describes that variant as being pathogenic in trans with another MUTYH variant. ${ }^{5}$ However, the definition of "DM?" in the HGMD suggests that the mutation is likely disease-causing (likely pathogenic). Interestingly, MUTYH p.Gln454Pro, the variant concurrently observed in the same individual in the primary article describing this mutation, is listed by the HGMD as "DM" (not "DM?") without any additional evidence. For a given $M U T Y H$ p.Gln267* variant, we can collect at least two pieces of evidence for pathogenicity, including the null variant and extremely low allele frequency. The variant could be classified as likely pathogenic according to the scoring rules of the American College of Medical Genetics and Genomics and the Association for Molecular Pathology guidelines for sequence interpretation. ${ }^{4}$ In addition, MUTYH pathogenic or likely pathogenic variants in our report were excluded from the estimation of the frequency of secondary findings because they were found in individuals who carry only one copy of the variant, causing recessive disease.

Classification of sequence variants to the appropriate category is very difficult. As the clinical use of exome or genome sequencing continues to rapidly increase, the importance of identification and reporting of secondary findings will be further emphasized. We believe that, although several limitations and weaknesses exist, our data provide evidence to support the genomic study of secondary findings in Korea. To improve the accuracy and consistency of variant classification, we must put in place stringent standards and guidelines and perform independent consensus reviews because there will always be the possibility of erroneous interpretation.

\section{DISCLOSURE}

The authors declare no conflict of interest.

\section{Mi-Ae Jang, $M D$, PhD ${ }^{1,2}$, Won Park, $B S^{3,4}$, Namshin Kim, $P h D^{3,4}$ and Chang-Seok Ki MD, PhD}

${ }^{1}$ Department of Laboratory Medicine and Genetics, Samsung Medical Center, Sungkyunkwan University School of Medicine, Seoul, South Korea; ${ }^{2}$ Current address: Department of Laboratory Medicine and Genetics, Soonchunhyang University Bucheon Hospital, Soonchunhyang University College of Medicine, Bucheon, South Korea; ${ }^{3}$ Epigenomics Research Center, Genome Institute, Korea Research Institute of Bioscience and Biotechnology, Daejeon, South Korea; ${ }^{4}$ Department of Functional Genomics, Korea University of Science and Technology, Daejeon, South Korea. Correspondence: Chang-Seok Ki (changski@skku.edu) or Namshin Kim (deepreds@kribb.re.kr)

\section{REFERENCES}

1. Biesecker LG. Overcalling secondary findings. Genet Med 2016;18:418.

2. Jang MA, Lee SH, Kim N, Ki CS. Frequency and spectrum of actionable pathogenic secondary findings in 196 Korean exomes. Genet Med 2015;17:1007-1011.

3. Robinson R, Carpenter D, Shaw MA, Halsall J, Hopkins P. Mutations in RYR1 in malignant hyperthermia and central core disease. Hum Mutat 2006;27: 977-989.

4. Richards S, Aziz N, Bale S, et al.; ACMG Laboratory Quality Assurance Committee. Standards and guidelines for the interpretation of sequence variants: a joint consensus recommendation of the American College of Medical Genetics and Genomics and the Association for Molecular Pathology. Genet Med 2015;17:405-424.

5. Kim DW, Kim IJ, Kang HC, et al.; Germline mutations of the MYH gene in Korean patients with multiple colorectal adenomas. Int J Colorectal Dis 2007;22:1173-1178

Advance online publication 10 March 2016. doi:10.1038/gim.2016.20 